\title{
The HIV epidemic in China: history, response, and challenge
}

\author{
$\mathrm{Na} \mathrm{HE}^{1, *}$, Roger DETELS ${ }^{2}$ \\ ${ }^{1}$ Department of Epidemiology, Fudan University School of Public Health, Shanghai 200032, China \\ ${ }^{2}$ Department of Epidemiology, UCLA School of Public Health, Los Angeles, CA 90095-1772, USA
}

\begin{abstract}
The first case of AIDS was reported in 1985 in China, but by the early 21 st century, the government estimated that there were 840,000 citizens living with HIV/AIDS. The number is increasing rapidly. The major risk groups are injection drug users (IDUSs; $43 \%$ ) and former plasma donors (27\%), but rates among heterosexual groups are rising rapidly. Sentinel surveillance was initiated in 1986, and now includes IDUs, men-who-have-sex-with-men, sexually transmitted disease clinic attendees, antenatal women, long-distance truck drivers, and sex workers. Although the government was slow to respond to the epidemic in the late 20th century, it has made a vigorous response in the early 21 st century. Components of that response include implementation and evaluation of harm reduction programs for IDUs, education to increase knowledge and reduce stigma, treatment and social support for rural and poor HIV/AIDS patients, widespread testing, and increased funding for HIV/AIDS programs. International agencies have been generous in their support of the government initiatives. To successfully combat the epidemic, China needs to develop and train the necessary infrastructure to implement its intervention programs, particularly in the rural areas, to vigorously combat stigma and discrimination, support research especially in the universities and research institutions other than the China Centers for Disease Control, develop a system for efficient exchange of research and program information, and update legislation to reflect the current situation.
\end{abstract}

Keywords: HIV/AIDS, China, epidemic.

\section{INTRODUCTION}

One-quarter of the world's population, 1.3 billion people, live in China. The majority of the population is Han Chinese $(91.9 \%)$, while the rest are a mix of different minority groups living primarily in southern and western China [1]. The GDP per capita is $\$ 5,600$ per year, but at least $10 \%$ of the population lives below the poverty line. Just less than $15 \%$ of the GDP is in agriculture, with the remainder in industry/construction and services. The total health expenditure (\$224 per capita) represents $5.5 \%$ of the GDP. The average life expectancy is 72 years. The eastern and urban parts of the country have experienced a rapid improvement in income and associated standards of living, with the adoption of a market economy in the 1990s, but the western areas of China are still suffering from high levels of poverty and poor quality of health services,

*Correspondence: $\mathrm{Na} \mathrm{HE}$

Tel/Fax: 86-21-54237519;

E-mail: nhe@shmu.edu.cn especially in the rural areas.

Although China has made great strides in controlling disease in the last 50 years, recently the country has been experiencing one of the most rapidly growing epidemics of HIV in the world. The first case of AIDS was reported in China in 1985, but the first outbreak was not recognized until 1989, when a cluster of infections was found among injection drug users (IDUs), mostly minorities, in villages in southern Yunnan [2, 3]. By 1993, HIV was reported to be occurring among plasma donors in the poorest provinces of central China [4]. By 1998, HIV was reported in all 31 provinces of China. The epidemic has now spread to commercial sex workers, among whom the prevalence has reached $3 \%$ in some areas, and to women attending antenatal clinics, among whom the prevalence is greater than $1 \%$ in some areas [5]. The HIV epidemic has concentrated in the rural areas in China that are least able to mount an effective response, a situation similar to that in other countries. This situation complicates intervention efforts and treatment of HIV/AIDS patients, and thus calls for innovative yet effective intervention strategies. 
Although the number of reported HIV cases was about 106,990 by the end of 2004, the officially estimated number of people living with HIV was 840,000 [6]. The HIV epidemic in China has already reached a phase of exponential growth. The number of reported infections has increased steadily by $30 \%$ each year from 1995 to 2000 $[7,8]$. In 2001, the increase in the reported number of HIV infections was $58 \%$, almost twice as high as in previous years [8]. In the ensuing years, the rate of HIV infection rate has continued to rise, reaching a $122 \%$ increase in the year 2002-2003 [8]. This illustrates that the number of reported HIV/AIDS cases in the country continues to increase steadily and significantly. Eighty per cent of HIV/ AIDS cases are among rural residents. More males (74\%) than females are infected, and three-quarters of those infected are 20 39 years old [9].

The majority of those infected has not been identified and do not know their disease status. This indicates an enormous gap in HIV testing and counseling in China, and poses a serious challenge to prevention and treatment, as well as care of HIV/AIDS, because a large number of patients remain to be identified and continue to spread the virus in the population. Clearly, if the epidemic is not contained in China, the social and economic impact will be devastating, not only to China, but also to the world.

\section{HIV/AIDS SURVEILLANCE}

HIV/AIDS surveillance was begun in 1986. In 1989, it became a legal requirement to report HIV-infected individuals. In 1995, the China Ministry of Health and the National Center for AIDS established 42 national sentinel sites in 23 of the 31 provinces, with the assistance of the World Health Organization (WHO). At that time, the target groups for sentinel surveillance included intravenous drug users (IDUs), patients attending sexually transmitted disease (STD) clinics, female commercial sex works, and long-distance truck drivers. Since 1997, women attending antenatal clinics (ANC) have been included in the sentinel surveillance. Former plasma donors (FPDs) were targeted by sentinel surveillance in 1998 and 1999, but not in the subsequent years. Since 2003, men who have sex with men (MSM) have also been recruited for HIV sentinel surveillance. By 2002, national sentinel sites had been implemented in 158 locations in all 31 provinces [8]. The number of national sentinel sites continued to rise to 194 by 2003 and 247 by 2004 . In addition, there had been more than 400 sentinel sites established by provincial Centers for Disease Prevention and Control that were funded by provincial governments [10]. Most recently, the Chinese National Center for AIDS, STDs \& TB has integrated the previously separate HIV/AIDS surveillance and STD surveillance into a comprehensive HIV/AIDS and STD surveillance system, to which behavioral surveillance has recently been added.

\section{RISK GROUPS}

There are three main types of high-risk activities facilitating HIV transmission in China: sharing of needles among IDUs, activities relating to blood products and blood transfusion, and unsafe sexual practices. Among reported HIV cases up to $2004,43.2 \%$ were IDUs and $26.8 \%$ were FPDs [10].

\section{Injection drug users}

Drug abuse has become a significant social and public health problem in China. While the reported number of drug users increased from 70,000 in 1990 to $1,050,000$ in 2003, a 15-fold increase [11], the actual number in 2000 was estimated to be 6 to 8 million [12].

The early epidemic among drug users was part of the epidemic occurring in the countries of the "Golden Triangle" (Thailand, Myanmar, northeastern India, and southern Yunnan) [2, 3]. Although HIV infection has been reported in all 31 mainland provinces, there is considerable variation in the transmission routes and infection rates. The epidemic began among IDUs in areas of Yunnan province bordering Myanmar, then spread among IDUs residing along major drug trafficking roads to Guangxi, Xinjiang, Sichuan, Guangdong, and other provinces $[13,14]$.

Among drug users, about half inject drugs, and progression from non-injecting to injecting drug use is common, largely because injecting is seen as a more costeffective way of experiencing the effects of the drug [15, 16]. According to sentinel surveillance data, the overall seroprevalence of HIV among IDUs in China is over 5\% [6]. In some areas in Guangxi, Xinjiang, Guizhou, Sichuan, Jiangxi and Yunnan, however, the seroprevalence of HIV among IDUs is as high as $50 \%$ to $80 \%$ [6]. The geographic environment and the large market for drugs make it very difficult to effectively eliminate and control drug use. Low awareness of HIV risk, a paucity of HIV/ AIDS knowledge, and the common practice of risky behaviors further aggravate the potential explosion of an HIV epidemic in this special high-risk group. Prevention of injecting drug use by young people will probably require recognition by and mobilization of the rural communities (in which the majority of drug use occurs) to address the problem [17].

In 2002, the National Center for AIDS implemented pilot methadone replacement programs and needle/syringe exchange programs in several areas with high proportions of IDUs. In 2005, the government made the decision to expand the number of methadone replacement programs and needle/syringe exchange programs to all areas of China 
with high numbers of IDUs [10]. This strategy should significantly reduce the prevalence of HIV among injecting drug users, although the needle/syringe programs will not reduce the prevalence of IDUs.

\section{Plasma donors}

An outbreak of HIV among commercial plasma donors in poor rural communities occurred in several provinces between 1994 and 1996 [4, 6, 18, 19; and observations of G JI, R DETELS, et al]. From the late 1980's to the early 1990 's, thousands of small commercial plasma collection stations were set up in rural areas by blood product companies. Because of deep-rooted cultural beliefs that make Chinese people in general reluctant to donate blood, blood products in China are mostly derived from blood donated by individuals who receive payment. At some local government-run blood banks and in many private underground blood banks operated in the early and middle 1990s in central China, blood was often collected from several villagers at the same time, and mixed together in a container or a centrifuge from which the plasma was collected. The remaining blood cells, which had been mixed with the cells of others, and all too often were contaminated with HIV, were returned to the donors. Such procedures, as well as recycling of used needles and inadequately sterilized equipment, allowed HIV to be rapidly transmitted among these donors, generating a large number of HIV-infected farmers and peasants. The seroprevalence of HIV among FPDs was $9.1 \%$ in one county studied and $17 \%$ in another, and was closely related to the frequency of plasma donations [20]. While HIV seroprevalence was $8.3 \%$ among the FPDs who on average made less than five donations per month, it was $16.1 \%$ among those having made more than five but less than 15 donations per month, and $41.7 \%$ among those having made more than 15 donations per month [19]. Although China has waged a vigorous battle against illegal blood product enterprises, the HIV epidemic has been established in several areas in central China, and is affecting a larger population through unprotected sexual intercourse with spouses and other sexual partners. The plasma collection centers were closed in 1995, however, many still operated illegally. When it became clear that a major source of HIV infection was through blood, screening of the blood supply was implemented in 1996. Until recently, many FPDs who may have been infected with HIV had never been tested. In a survey of HIV infection among 297 FPDs, the prevalence was 74\% [21]. In 2000, 20 hospitalized FPDs were found to be HIV-positive and had developed AIDS. None had been found to be HIV-positive before hospitalization. Therefore, the actual magnitude of the HIV epidemic among FPDs may be much greater than indi- cated by current statistics. Statistics are not available for the number of plasma and blood recipients who may have been infected by the contaminated blood products.

\section{Heterosexual transmission}

Sexual behavior in China has changed dramatically over the last two decades. With the shift to a market economy in the early 1990s, the disparities between rich and poor increased. Wealthy entrepreneurs became able to buy sex, and those unable to adapt to the new system became poor. Many poor women turned to commercial sex as the demand for these services increased. Commensurate with the rise in the number of individuals having multiple partners, there was a rapid increase in the prevalence of sexually transmitted diseases (STDs). Between 1990 and 1998 , the reported prevalence of syphilis increased nearly 20 -fold, and the incidence of gonorrhea increased threefold $[22,23]$. In a nationwide survey, Parrish et al reported that $14.6 \%$ of high-income men had had sex with sex workers who were infected with chlamydia [24]. In a study of market vendors in Fuzhou, Detels et al reported that in 2002 the prevalence of any STD was $18 \%$ [25]. Even in rural areas, having multiple partners became increasingly common [26]. The sharp rise in the number of migrants engaging in extramarital sex has also increased. In a study of migrants to Shanghai, He et al reported STD rates of $3.2 \%$ in construction workers and $5.6 \%$ for factory workers and market vendors [27].

In 2004, the overall prevalence of HIV among commercial sex workers in sentinel surveillance sites was $1 \%$, and in some areas, was as high as 6.7\% [6]. In Yunnan and Guangxi, increasing proportions of injecting drug users are females, most of whom are also sex workers (Detels, personal communication). Thus, they are at risk for HIV infection both from drug use and from commercial sex, and therefore act as a bridge from the high-risk groups to the general population of men with extramarital partners and their spouses. In a survey of sex workers in Guangxi, one-quarter had clients who did not use condoms, and half had clients who used them only sometimes [28].

\section{Men who have sex with men (MSM)}

China is now facing the challenge of the potential spread of the HIV/AIDS epidemic from high-risk groups to the general population through homosexual activities, particularly commercial sex activities. HIV prevalences of $1.3 \%$ to $17.7 \%$ have been reported in several surveys [29-31]. However, the MSM population in China tends to be hidden. Thus, these surveys have not been able to recruit representative samples. Further, many MSM are married and continue to have sexual relations with their spouses [32, 
33]. The size of the MSM population in China is unknown, but as long as they continue to engage in sex with both males and females, they can spread the epidemic.

\section{CHINA'S RESPONSE}

The government of China was initially slow to respond to the epidemic. Passive surveillance was initiated in 1985, but active surveillance was not initiated until 1995. In 1999, comprehensive surveillance, including eliciting of epidemiologic, serologic and behavioral information, was initiated to be carried out twice a year. The following sentinel groups are included in active surveillance, although not all groups are included in all the sentinel sites: STD clinic attendees, commercial sex workers, injection drug users (IDUs), long-distance truck drivers, women attending antenatal clinics, and MSM. Forty-three percent of cumulative HIV cases reported by 2004 were in IDUs, and $26.8 \%$ in FPDs. Thirty-five percent of cumulative HIV/AIDS cases are reported among 20-29 year-olds, and an equal proportion among 30-39 year-olds. The male: female ratio is 2.6:1. The majority of HIV transmission originated and continues to occur in the rural areas where treatment facilities and reporting mechanisms are weakest.

In 1988, China banned all HIV-infected foreigners from entering the country, but did not declare AIDS to be a reportable disease until 1991. At that time, they attempted to quarantine all AIDS patients. In 1995, China required premarital health examinations including testing for HIV [34]. In 1998, China conducted an international workshop on control of HIV/AIDS, which included both Chinese and foreign leaders. As a result of the workshop, the Law of Blood Donation was implemented, which called for tight regulation of all blood and plasma collection sites. The law, however, is not closely observed in rural areas [observations of Z WU, P FIGUEROA and R DETELS].

Since 1998, China has taken increasingly aggressive action to slow the epidemic. In 1998, the government developed and implemented medium- and long-term programs (1998-2010) for the prevention and control of AIDS. In 2001, it implemented the China HIV/AIDS Containment, Prevention and Control Action Plan (2001-2005), and a notice on strengthening HIV/AIDS prevention and control was issued by the highest government body in China, the State Council. In 2003, the premier and president visited AIDS patients in hospitals subsequently. Their appearance underscored the government's recognition of the seriousness of the epidemic and the commitment of the government to controlling it. In July 2004, Premier Jia Bao WEN signed a proclamation outlining the Chinese government's new initiatives in response to the HIV/AIDS epidemic. This proclamation was followed by increased funding and re- source mobilization for the fight against HIV/AIDS. In February 2004, the State Council established the HIV/AIDS Working Committee, with the Vice-Premier and the Minister of Health as directors. The committee includes viceministers from $23 \mathrm{key}$ ministries and vice-governors from the seven most affected provinces. In September 2003, the "Four Frees and One Care" policy was implemented, which provided free antiretroviral drugs to AIDS patients in rural areas and those with financial difficulties living in urban areas, free voluntary counseling and testing for HIV, free drugs for HIV-infected pregnant women to reduce mother to child transmission, HIV testing of newborn babies, free schooling for children orphaned by AIDS, and care and economic assistance to the households of people living with HIV/AIDS.

In March 2003, the China Comprehensive AIDS Response ("China CARES") was implemented. This program, to initiate a comprehensive mechanism for HIV/AIDS prevention and treatment, provides for free HIV testing and treatment of all infected individuals in 127 severely affected counties within 31 provinces except for the Beijing, Shanghai and Tianjin municipalities. The 127 counties were selected during two years, with 51 counties selected for implementation in 2003 and 76 counties in 2004. The national government grants each of the 127 counties $\$ 40,000-75,000$ per year. This amount is not sufficient, and the counties also need to contribute to the implementation of the program in their areas. The scope of the China CARES program differs among areas, but the government is attempting to provide standard procedures across the 127 counties. The China AIDS Roadmap Tactical Support Project provides $\$ 10$ million for multisectoral mobilization, leadership building, information exchange, methadone maintenance programs, and clean needle exchange programs. Through June 2005, over 100 methadone clinics have been opened in areas with high levels of HIV/AIDS among IDUs. Trainees of the UCLA/Fogarty AIDS International Training and Research Program are assisting the China Centers for Disease Control in comparing voluntary counseling and testing centers in the China CARES counties and other areas and evaluating the methadone clinics.

\section{GLOBAL FUND PROGRAM}

Since 2004, the Global Fund to Fight AIDS, Tuberculosis, and Malaria has been working complementary to China CARES. The Global Fund program enhances training for local health workers, and provides antiretroviral treatment (ART), treatment for opportunistic infections, VCT, condom promotion, and social support. The program began with a baseline survey at the end of 2004. Meanwhile, a public health campaign was conducted around the time of World AIDS day, targeting students and villagers to im- 
prove HIV basic knowledge. To closely cooperate with the China CARES program, the Global Fund program provides additional funding to facilitate implementation of China CARES. It provides a subsidy to local health workers who are in charge of the ART program at the community level, a subsidy to HIV-positive villagers who are severely impoverished, and procures essential vehicles and equipment.

China has received funds to combat the HIV/AIDS epidemic from the Global Fund, the Global AIDS Program, several United Nations Agencies, USAID (primarily through Family Health International), the U.S. Centers for Disease Control, and the Global AIDS Program. In addition, China was the first country to be awarded a Comprehensive International Program of Research on AIDS (CIPRA) grant from the National Institutes of Health.

\section{FUTURE CHALLENGES}

China has a huge challenge ahead in containing the HIV/ AIDS epidemic and treating the million or more people already infected with HIV. China has implemented ambitious programs to slow the epidemic and treat infected individuals, but lacks trained manpower, particularly at the rural level, to effectively implement these intervention programs and to treat infected individuals.

\section{Stigma and discrimination}

Although the Chinese government has implemented a number of massive health education programs about HIV/ AIDS in the media, the stigma associated with HIV/AIDS and related risk behaviors is still a major problem in China. In both rural and urban areas, discrimination towards people living with HIV/AIDS (PLWHA) and their families is common, and is correlated with low willingness to participate in HIV/STD prevention and intervention activities [35]. Stigma is a major barrier to being tested for HIV and disclosing HIV infection status. Although China may require HIV testing in areas known to have high rates of HIV infection, stigma will remain a barrier in other areas, and will cause many Chinese to avoid testing and the social consequences of testing, unless effective programs to reduce stigma are implemented. As a result, many HIVinfected persons will continue to be unaware of their infection status, and will unknowingly continue to infect others, spreading the epidemic further. Thus, studies to identify major factors associated with stigma and discrimination towards HIV/AIDS and evidence-based prevention and intervention programs for reducing HIV/AIDS-related stigma in China are urgently needed. Such interventions may need to take into account community characteristics, such as level of HIV/AIDS-related risk behaviors and level of development in the community [36].

\section{Limited human resources}

After two decades of efforts to control HIV/AIDS, it is now more evident than ever before that limited availability of well-trained health workers or health professionals is the most severe barrier to successfully implementing HIV/ AIDS prevention and control programs. Human resources must be significantly increased in the near future in China. In the meantime, AIDS patients who need ART and medical care are cared for by inexperienced health professionals at the provincial, prefectural and county levels. Therefore, further training at all levels should be a priority for China in the coming years. Moreover, since many HIVinfected individuals in China are living in rural and remote areas that are relatively less developed, more human resources and training programs are required and needed for rural China.

\section{Resource allocation}

In the foreseeable future, obtaining adequate resources for HIV/AIDS control will continue to be a real challenge for China. Effectively allocating the limited resources into areas that need them the most requires scientific assessment and careful consideration. Not all the funds should be earmarked for treatment; some resources need to be allocated for mass media programs of HIV/AIDS education, destigmatization efforts, HIV counseling and testing, behavioral intervention, harm reduction programs, and training of health professionals.

\section{HIV/AIDS research}

HIV/AIDS research in China is not keeping pace with the rapid spread of the disease. During the past two decades, the China CDC has been responsible for both research and control of HIV/AIDS. Unfortunately, universities and other research institutions, including the Chinese Academy of Science, have not been actively involved in national efforts for HIV/AIDS control, and have received little funding for HIV/AIDS research. As the China CDC complains that it is overburdened and lacks adequate human resources for HIV/AIDS control and research, they should utilize the universities and institutions that are eager to have more funding for HIV/AIDS research and to have more opportunities to participate in HIV/AIDS activities. Universities have many well-educated faculty members and students who can play a key role in HIV/ AIDS research. Thus, it is crucial for China to encourage and support these institutions to conduct HIV/AIDS research.

Given the fact that an HIV and/or AIDS vaccine will not be available in the coming years and the currently available antiviral drugs cannot completely eliminate HIV, behavioral intervention is the most effective way to prevent 
the spread of the HIV/AIDS epidemic. Therefore, it is important for China to support more operational research, which is usually policy-oriented. These projects should be designed to look for the main determinants for HIV transmission in China, to identify cost-effective prevention/behavioral intervention strategies, to better understand the HIV/AIDS-related health needs of vulnerable populations, to provide better health services to them, to develop a friendly and supportive environment for HIV/ AIDS patients, to evaluate the massive programs that China has already implemented to avoid persistence of ineffective programs, and to identify barriers to successful implementation.

\section{Information sharing and program integration}

Researchers, public health practitioners, officials, and the general population find it very difficult to obtain access to national and/or provincial HIV/AIDS and STD surveillance data. China has not set up a mechanism of nationwide data sharing and information exchange for HIV/ AIDS control. Thus, much useful information and data are not being utilized by governments and organizations in designing and conducting programs. Consequently, experience and lessons gained from one program might not be learned by other programs, and many HIV/AIDS prevention and control programs conducted by different institutions or organizations are likely to overlap with each other. This wastes resources that could have been applied to other programs. For various reasons, many HIV/AIDS prevention and control programs are undertaken simultaneously with overlapping funding from various resources, including both government and non-government organizations and both national and international sources. It is not uncommon that data collection in local CDCs are not analyzed and utilized for guiding subsequent control measures.

Thus, to prevent waste of resources and time and ineffective use of collected data, China needs to establish a multi-sector network to integrate various resources and various programs in an overall plan framework. China also needs to establish guidelines and protocols for data collection, data analysis, and information sharing and utilization at all levels.

\section{HIV/AIDS control among migrants}

China is the most populous country in the world, and has more than 120 million peasant migrants who have left their home villages for urban areas. The floating status of migrants may facilitate HIV spread from one area to another, as migrants often engage in risk behaviors [25, 37]. A number of migration-related factors have been demonstrated to increase the likelihood of an HIV epidemic among and beyond migrants, including low social and economic status, limited education, little knowledge of condom use and HIV/STDs, low awareness of HIV/AIDS, separation from families, as well as economical, linguistic, and geographical barriers to health services. Migrants are no longer subject to attitudes and social norms that previously guided their behavior in their rural families, communities, and cultures. Long-term separations from their home environment may encourage migrants to engage in risky behaviors that they would not otherwise have engaged in. In some settings, such as long-distance truck services and construction sites in China, living and recreational environments for migrants and mobile workers are almost exclusively male, which leads to the development of commercial sex services and the pressure to use them. Due to limited access to health care, migrants are more likely than urban residents to remain untreated or treated inappropriately after contracting an STD. Inappropriate treatment of STDs makes individuals more vulnerable to HIV infection and more likely to transmit HIV once infected. Preventing HIV transmission within and beyond migrant groups has become one of the most serious public health challenges that China must address. HIV/AIDS prevention and control programs specifically designed for migrants are urgently needed.

Communities that accept migrants need to adopt health policies and practices that will remove economic, administrative, and linguistic barriers to migrants' access to health care and, in particular, HIV/STD-related health care. These policies should specifically encompass all types of migrants, including the most vulnerable, such as commercial sex workers and undocumented migrants. Gender issues will also need to be integrated into policies and programs. In the meantime, successful HIV/AIDS prevention and control programs for migrant populations tend to be those developed with and guided by migrant communities and involve substantial community input.

\section{Dilemma of HIV/AIDS control among illegal popula- tions}

Needle sharing among IDUs and unprotected sexual behaviors with commercial sex workers have driven the HIV/AIDS epidemic in China. Both behaviors are illegal and intolerable to the Chinese government. Therefore, those who engage in such illegal behaviors are subject to strict punishment. To avoid being punished or put in jail, drug users and commercial sex workers and their employers have had to choose to stay underground. This makes it extremely difficult to approach and work with them. However, to effectively control the spread of the HIV/ AIDS epidemic, the government must implement HIV/AIDS prevention and intervention programs among these groups. 
Successful implementation of such programs requires the government to accept the existence of such behaviors and to accept and closely collaborate with those who are engaging in these behaviors. In this regard, public health workers must spend time and resources to approach them, gain their trust, and change their behaviors. Unfortunately, the government periodically asks the police to apprehend individuals in these groups, fine them, shut down their venues or establishments, or even put them in jail. Therefore, when public health workers want to work with these people on HIV/AIDS prevention and intervention, they are not welcomed. There is fear that public health workers will reveal their identity and illegal behaviors to the police. This has created many difficulties and dilemmas for implementation of HIV/AIDS control programs targeting these groups. China would be more successful in controlling HIV in these groups if they monitored their activities rather than futilely attempting to suppress them.

\section{Legislation}

Over the last 20 years, laws and regulations regarding HIV/AIDS have been issued. However, the rapid spread of HIV has underscored the limitations of these laws and regulations. Thus, there is a need to revise, modify and update them to reflect the current situation and experience with various prevention and intervention strategies that have been implemented and evaluated. An example of the approach that is needed has been the action of the Shanghai government, which has organized lawyers, law experts, HIV/AIDS researchers, medical practitioners, and sociologists to discuss and develop new regulations for HIV/ AIDS prevention/control and care for AIDS patients. This also needs to be done at the local, provincial, and national levels for China.

China also needs to consider what approach to take for control of the HIV/AIDS epidemic. Up to now, they have tried to suppress the activities that promote exposure to HIV/AIDS, including commercial sex. Their recent decision to implement methadone replacement and needle exchange programs is an example of their recognition of the difficulty of eliminating drug use and the overriding need to reduce HIV transmission within the drug-using population and from them to the general population. A similar approach should be considered for reducing the risk of heterosexual transmission through commercial sex workers. Eliminating commercial sex in the current climate of capitalism is unlikely to succeed. Making commercial sex safe, however, can effectively reduce STDs, including HIV.

\section{ACKNOWLEDGEMENT}

This research was supported by NIH/Fogarty Interna- tional Center grant D43 TW000013 (RD).

\section{REFERENCES}

1 China Census Bureau. The Tenth China National Census, Beijing, China Census Bureau: Beijing 2001.

2 Ma Y, Li Z, Zhang K, et al. HIV was first discovered among IDUs in China. Chin J Epidemiol 1990; 11:184-5.

3 Zhang JP, Chen HH, Jia MH, Zhang Y, Epidemic of HIV infection and prevention research in Yunnan, 1989-98. Chin J Epidemiol 1999; 20:377-80.

4 Wu Z, Detels R. HIV-1 infection in commercial plasma donors in China. Lancet 1995; 346:61-2.

5 Qi XQ. HIV/AIDS epidemiology and China five-year action plan and research. Beijing, China: China-United States Workshop on HIV/AIDS Research, 2002.

6 China Ministry of Health and UN Theme Group on HIV/AIDS in China. A Joint Assessment of HIV/AIDS Prevention, Treatment, and Care in China: Beijing 2004.

7 China Ministry of Health and UN Theme Group on HIV/AIDS in China: Beijing 2003.

8 China Center for Disease Control \& Prevention. HIV/AIDS Surveillance Report: Beijing 2004.

9 Zheng X. Strengthening national capability of surveillance on AIDS and STDs. Chin J Prevent Med 2001; 2:3-4.

10 Wang LD. HIV/AIDS Prevention and Control in China. 7th International Conference on AIDS in Asia and the Pacific. Kobe, Japan; July 2005.

11 Office of the National Narcotic Control Committee. Annual Report on Drug Control in China, 2003.

12 World Health Organization. Report on the status of HIV and AIDS in China, 2001.

13 Sun X, Nan J, Guo Q. AIDS and HIV infection in China. AIDS 1994; 8 (Suppl 2):S55.

14 Wu ZY. Epidemics of HIV/AIDS in China. Science Press: Beijing 1999:39-56.

$15 \mathrm{Wu}$ Z, Detels R, Zhang J, et al. Risk factors for intravenous drug use and sharing equipment among young male drug users in southwest China. AIDS 1996; 10:1017-24.

$16 \mathrm{Wu} \mathrm{Z}$, Zhang J, Detels R, et al. Risk factors for initiation of drug use among young males in southwest China. Addiction 1996; 91: 1675-85.

$17 \mathrm{Wu}$ Z, Detels R, Zhang J, Li V, Li J. Community-based trial to prevent drug use in Yunnan, China. Am J Publ Hlth 2002; 92: 1952-7.

18 Zheng X, Wang Z, Xu J, et al. The epidemiological study of HIV infection among paid plasma donors in one county of China. Chin J Epidemiol 2000; 21:253-5.

$19 \mathrm{Wu}$ Z, Rou K, Detels R. Prevalence of HIV infection among former commercial plasma donors in rural eastern China. Hlth Policy Plan 2001; 16:41-6.

20 China Center for Disease Control \& Prevention, National Center for AIDS/STD Control \& Prevention, 2003.

21 Liu SZ, Zheng XW, Fu JH, et al. HIV prevalence and subtypes among paid blood donors in Shangdong and Hubei Provinces, China. Chin J Prevent Med 2001; 2:8-11.

22 Chen XS, Gong XD, Liang GJ, Zhang GC. Epidemiologic trends of sexually transmitted diseases in China. Sex Transm Dis 2000; 27:138-42.

23 Cohen MS, Ping G, Fox K, Henderson GE. Sexually transmitted 
diseases in the People's Republic of China in Y2K: back to the future. Sex Transm Dis 2000; 27:143-5.

24 Parish WL, Laumann EO, Cohen MS, et al. Population-based study of chlamydial infection in China: a hidden epidemic. JAMA 2003; 289:1265-73.

25 Detels R, Wu Z, Rotheram MJ, et al. Sexually transmitted disease prevalence and characteristics of market vendors in eastern China. Sex Transm Dis 2003; 30:803-8.

26 Liu HJ, Xie J, Yu WZ, et al. A study of sexual behavior among rural residents of China. J AIDS Human Retrovirol 1998; 19:808.

27 He N, Detels R, Zhu JD, et al. Characteristics and sexually transmitted diseases of male rural migrants in a metropolitan area of eastern China. Sex Transm Dis 2005; 32:286-92.

28 China CDC, National Center for AIDS/STD Control and Prevention. Annual Report on HIV/AIDS Epidemic in China in 2002: Beijing, 2003.

29 van den Hoek A, Yuliang F, Dukers NH, et al. High prevalence of syphilis and other sexually transmitted diseases among sex workers in China: potential for fast spread of HIV. AIDS 2001; 15: 753-9.

30 Zhang BC, Li XF, Shi TX, Cao NX, Hu TZ. Survey of the high risk behaviors and other AIDS/STI related factors among men who have sex with men (MSM) in mainland China. Chin J Dermatol 2002; 35:214-7.

31 Qu SQ, Zhang DP, Wu YH, Zhu H, Zheng XW. Seroprevalance of HIV and risk behaviors among men who have sex with men in a northeast city of China. Chin J STD/AIDS Prevent Cont 2002; 8:145-8.

32 Choi K, Liu H, Guo Y, et al. Emerging HIV - an epidemic in China in men who have sex with men. Lancet 2003; 361:2125-6.

33 Zhang BC. Current situation of MSM in China and HIV intervention. J Prevent Med Infect 2003; 19 (Suppl):1-2.

34. Wu Z, Rou K, Xu C, Lou W, Detels R. Evaluation of premarital HIV/AIDS counseling with voluntary HIV testing when applying for a marriage license in China. AIDS Educ Prevent 2005; 17: 12-21.

35. Yang H, Li X, Stanton B, et al. Willingness to participate in HIV/ STD prevention activities among Chinese rural-to-urban migrants. AIDS Educ Prev 2004; 16:557-70.

36. Chen J, Choe MK, Chen S, Zhang S. Community environment and HIV/AIDS-related stigma in China. AIDS Educ Prevent 2005; 17:1-11.

37. He N, Detels R, Chen Z, et al. Sexual behavior among employed male rural migrants in Shanghai. AIDS Educ Prevent 2005; in press. 